\title{
Anticoagulation of Behcet's Disease with Thrombosis: A Series of 28 Cases in a Tertiary Care Centre in Saudi Arabia
}

\author{
Muneerah Albugami ${ }^{1}$, Ahmed Al Shaikh ${ }^{1}$, Abdulaziz Alrashed ${ }^{1}$, Hanan Al Hamzi ${ }^{1}$, \\ Alanoud Khuthaila ${ }^{2}$, Abdelazeim Elamin ${ }^{1}$, Mumin Siddiquee ${ }^{1}$, Usamah El Alem ${ }^{1}$, \\ Lama Amer ${ }^{1}$, Yasmin Altwaijri ${ }^{2}$
}

${ }^{1}$ Medicine Department, King Faisal Specialist Hospital and Research Centre, Riyadh, Saudi Arabia

${ }^{2}$ Research Centre, King Faisal Specialist Hospital and Research Centre, Riyadh, Saudi Arabia

\section{Email address:}

Drmb99@yahoo.com (M. Albugami), alshaikh@kfshrc.edu.sa (A. Al Shaikh), aalrashed03@kfshrc.edu.sa (A. Alrashed), alhamzih@yahoo.com (H. Al Hamzi), alanoudk@kfshrc.edu.sa (A. Khuthaila), abdelazeim@hotmail.com (A. Elamin), msiddiquee@kfshrc.edu.sa (M. Siddiquee), Usamah El Alem (U. El Alem), laamer@kfshrc.edu.sa (L. Amer), yasmin@kfshrc.edu.sa (Y. Altwaijri)

\section{To cite this article:}

Muneerah Albugami, Ahmed Al Shaikh, Abdulaziz Alrashed, Hanan Al Hamzi, Alanoud Khuthaila, Abdelazeim Elamin, Mumin Siddiquee, Usamah El Alem, Lama Amer, Yasmin Altwaijri. Anticoagulation of Behcet's Disease with Thrombosis: A Series of 28 Cases in a Tertiary Care Centre in Saudi Arabia. American Journal of Internal Medicine. Vol. 5, No. 4, 2017, pp. 57-60. doi: 10.11648/j.ajim.20170504.12

Received: March 30, 2017; Accepted: April 24, 2017; Published: June 15, 2017

\begin{abstract}
Behcet's disease (BD) is a multisystemic inflammatory disorder of unknown etiology. There is few data about BD in Saudi Arabia. This is a retrospective chart review study of 28 patients of BD with thrombosis on long term anticoagulation. A total of 28 BD patients, their mean age was 43.8 (25-60) years. The mean age of first thrombosis was 29.2 (14-42) years. Provoked thrombosis was reported in $17.86 \%$ of the patients. The commonest site of thrombosis was superficial femoral vein $35.71 \%$, followed by popliteal vein 32.14\%, pulmonary embolism (PE) 32.14\%. Anti B2 glycoprotein 1 antibodies was identified in $31.82 \%$ of patients, Anti cardiolipin Antibodies in $22.73 \%$, Leiden factor V in $18.18 \%$. Activity of the disease during thrombosis was recognized as oral ulcer $44.44 \%$, genital ulcer $33.33 \%$, Human leukocyte antigen-B (HLAB) was $32.14 \%$. Thrombosis while patient on anticoagulation was reported in $32.14 \%$ of patients. Bleeding was reported in $14.29 \%$ of patients, common site of bleeding was gastrointestinal bleeding. Survival rate was 78.57\% and 2 patients were died (7.14\%) due to malignancy. Conclusion: Thrombosis in BD patients is male predominance, more in venous, has low mortality, frequency of thrombosis is more in the presence of thrombophilia and active BD. The prevalence of thrombophilia among BD patients is high. The presence of thrombophilia still affect the physician decision regarding the initiation and duration of anticoagulation in BD. The incidence of bleeding with long term anticoagulation is low. Further research is needed to find out the prevalence of cancer among BD in Saudis.
\end{abstract}

Keywords: Behcet's Disease, Anticoagulation, Trombosis, Thrombophilia

\section{Introduction}

Behcet's disease (BD) is a systemic disease characterized by mucocutaneous, ocular, vascular and neurologic manifestations. The exact pathophysiology of $\mathrm{BD}$ remains unknown. Human leukocyte antigen-B51 (HLA-B51) is associated with $\mathrm{BD}$ in $70 \%$ to $80 \%$ of patients, especially in regions where the disease is more prevalent [1]. A novel HLAB antigen was identified in a Saudi family affected with BD. This antigen was named as HLAB4202 and its frequency in the Saudi population was estimated to be around $10 \%$ [2]. Vascular involvement in $\mathrm{BD}$ is presenting as venous and arterial thrombosis and as aneurysms. Vascular involvement is one of the major causes of morbidity and mortality in BD. Pulmonary artery aneurysm carries a high mortality of approximately $25 \%$ [3]. The therapeutic approach towards thrombosis in BD differs significantly among rheumatologists from different countries [4]. There is 
few data about BD in Saudi Arabia [5]. The objective of this study is to report our experience in long term anticoagulation of $\mathrm{BD}$ with thrombosis.

\section{Methods}

A retrospective, cohort study utilized chart review of 28 patients of BD with thrombosis on long term anticoagulation. Patients were identified from the registry of thromboembolic disorders at King Faisal Specialist Hospital and Research Centre (KFSH\&RC). All adults (age $>14$ years) BD patients had thrombosis followed up in the anticoagulation clinic at KFSH\&RC were included in the study.

\section{Statistical Analysis}

For this study, all the statistical analysis of data was done by using the software package SAS version 9.4 (SAS Institute Inc., Cary, NC, USA). Descriptive statistics for the continuous variables are reported as mean \pm standard deviation and categorical variables are summarized as frequencies and percentages.

\section{Result}

A total of $28 \mathrm{BD}$ patients ( 21 males, 7 females) developed thrombosis and their mean age was 43.8 (25-60) years. The mean age of first thrombosis was 29.2 (14-42) years. Provoked thrombosis was reported in $17.86 \%$ of the patients. The commonest site of thrombosis was superficial femoral vein $35.71 \%$, followed by popliteal vein $32.14 \%$, pulmonary embolism (PE) 32.14\%. Massive PE with hypotension reported in one patient, sagittal sinus thrombosis $17.86 \%$, right ulnar Artery thrombosis $16.67 \%$, iliac veins thrombosis $16.67 \%$, superior mesenteric artery thrombosis $16.67 \%$, Budd Chiari Syndrome (portal /hepatic vein) thrombosis $10.71 \%$, cerebral vein thrombosis $14.29 \%$, transverse sinus thrombosis $7.14 \%$, Mesenteric vein thrombosis $3.57 \%$, Inferior vena cava thrombosis $3.57 \%$, No thrombosis reported in axillary vein, brachial vein, cavernous sinus, jugular vein internal or external, and superior vena cava. Three patients of PE had either superficial femoral vein or popliteal vein thrombosis. However $21.4 \%$ had PE without evidence of lower extremities deep vein thrombosis. Thrombophilia workup was done in $78.57 \%$ of patients. Anti B2 glycoprotein 1 antibodies was identified in $31.82 \%$ of patients, Anti cardiolipin Antibodies in $22.73 \%$, Leiden_factor $\mathrm{V}$ in $18.18 \%$, Lupus anticoagulant in $9.09 \%$, MTHFR mutations in $13.64 \%$, the result was negative for protein $\mathrm{C}$ deficiency, protein $\mathrm{S}$ deficiency, activated protein $\mathrm{C}$ resistance, antithrombin III deficiency, paroxysmal nocturnal hemoglobin, homocysteine and prothrombin gene mutations. Activity of the disease during thrombosis was recognized as oral ulcer $44.44 \%$, genital ulcer $33.33 \%$, uveitis $11.11 \%$, arthritis $14.81 \%$, rash $18.52 \%$, HLA B $32.14 \%$, high erythrocyte sedimentation rate (ESR) $18.52 \%$, high C-reactive protein (CRP) $22.22 \%$, and low hemoglobin
$11.11 \%$. Thrombosis while patient on anticoagulation was reported in $32.14 \%$ of patients and $77.78 \%$ of patients were compliant to warfarin. The international normalized ratio (INR) mean was 2.6 (therapeutic). Complication of anticoagulation was bleeding reported in $14.29 \%$, common site of bleeding was gastrointestinal bleeding; it was reported in 3 patients. Pulmonary hemorrhage was reported in one case, no cerebral bleeding or Intra-abdominal bleeding was reported. Outcome: 22 patients $(78.57 \%)$ were still alive and 2 were dead (7.14\%). The outcomes of 4 patients were unknown. The cause of death in 2 cases was malignancy (ovarian adenocarcinoma stage 4). No reported death due to infection or cardiac or bleeding.

\section{Discussion}

In present study the mean age was 43.8 years. In most of the studies the average age of BD was $29.25 \pm 14.25$ years. The onset occurred in the third or fourth decade of life $[6,7]$. Majority of our patients were male and the mean age of first thrombosis was 29.2 years. One study showed that age at first thrombotic events was $33 \pm 10$ years old [8]. Vascular thrombosis occurred more in male patients. Male sex and a younger age of onset usually associated with more severe diseases [9].

The vascular manifestations were reported in $10-40 \%$ of cases of $\mathrm{BD}$ and venous involvement was more common $80 \%$ to $90 \%$ of cases $[10,11] .57 \%$ of our patients had unprovoked thrombosis. It is predominantly venous thrombosis and the commonest site of thrombosis was superficial femoral vein followed by popliteal vein. PE was reported in $32.14 \%$ of our patients. $21.4 \%$ of patients had PE without evidence of lower extremities thrombosis. Previous studies showed that deep vein thrombosis (DVT) and superficial vein thrombophlebitis of lower extremities were the typical manifestations, but thrombosis may occur anywhere in the venous system $[12,13]$. In $\mathrm{BD}$ thromboembolism could be rare, maybe due to the high adherence of thrombi to the diseased veins [14]. Among our patients the arterial thrombosis was uncommon. In previous Saudi study DVT was diagnosed in $25 \%$ and arterial thrombosis in $18 \%$ of BD patients [5].

The cause of vascular thrombosis in BD could be related to endothelial cell injury or pathological activation due to vasculitis. However, inherited prothrombotic conditions, such as factor $\mathrm{V}$ Leiden mutation, prothrombin gene mutation, protein $\mathrm{C}$, and $\mathrm{S}$ deficiency may result in the development of thrombosis as well. In the present study thrombophilia workup was done in $78.57 \%$ of BD patients. Risk factors for thrombosis were identified like Factor V Leiden (FVL), Anti B2 glycoprotein 1 antibodies, Anti cardiolipin Antibodies, Lupus anticoagulant and MTHFR mutations. FVL was identified in $30-37.5 \%$ of BD patients from Turkey and Saudi Arabia [13]. The prevalence of thrombophilia among our BD patients was high and the prevalence of Anti B2 glycoprotein 1 antibodies and Anti cardiolipin Antibodies were more than FVL. Antiphospholipid antibodies promote 
coagulation, these antibodies was associated with arterial and venous thromboembolism. Many antiphospholipid antibodies were tested in BD. Elevated levels of anticardiolipin antibodies were associated with retinal and cerebrovascular pathology in BD [15]. In study from Turkey, among 96 patients with thrombosis, patients with recurrent thrombotic events had a higher incidence of thrombophilias than those patients with only single thrombotic event [16]. The presence of thrombophilia most likely affected the physician decision regarding the initiation and duration of anticoagulation in BD patients [17].

The European League against Rheumatism (EULAR) guidelines discourage the use of anticoagulants and antiplatelet agents, both on account of the increased risk of bleeding and because protection from pulmonary embolism is seen as a low priority. The therapeutic approach towards thrombosis in BD differs significantly among rheumatologists from different countries [4]. A retrospective study of 936 patients with BD from 15 rheumatology clinics in Turkey was conducted. The researchers concluded no additional benefits were observed with treatment of Vascular BD with anticoagulants [18].

Activity of $\mathrm{BD}$ was confirmed during thrombosis and identified as oral ulcer, genital ulcer, uveitis, arthritis, rash, HLA B, High ESR, High CRP and Low HB. So ongoing thrombosis is most likely related to the activity of disease and it was not affected by anticoagulation treatment. The main factor responsible for the increased frequency of thrombosis in $\mathrm{BD}$ is thought to be endothelial damage/activation, abnormal fibrinolysis and altered platelet function. All had been associated with the development of thrombosis in BD [8]. The relationship between inflammation and thrombosis had been deeply investigated. Inflammation-induced thrombosis was considered a feature of BD especially during active disease. The pathophysiology of thrombosis in BD is unknown, but systemic inflammation seemed to play a major role whereas other thrombophilia factors were less relevant [19].

Our BD patients had low incidence of bleeding on long term anticoagulation. Gastrointestinal bleeding was the commonest site of bleeding. Pulmonary hemorrhage was rare, no cerebral or Intra-abdominal bleeding was reported. In this study BD patients with thrombosis had low mortality rate. In a 20-year follow-up of 387 Turkish patients with BD, most were doing better at 20 years [20]. The mortality in a cohort of 817 patients in France was 5\% at a median follow-up of 7.7 years [21]. BD Survival was improved as the result of earlier recognition and treatment using immunosuppressive agents [20, 21].

Only two female patients died of malignancy (ovarian adenocarcinoma stage 4). There were few case reports and case series regarding the association between malignant diseases and BD in the literature. Both hematologic and solid malignancies had been reported [22]. Female gender, older age and gastrointestinal tract involvement were more among patients with malignancy. Longer disease duration was more seen in BD patients with solid neoplasms [23].

\section{Conclusion}

Thrombosis in BD patients is male predominance, more in venous, has low mortality, frequency of thrombosis is more in the presence of thrombophilia and active BD. The prevalence of thrombophilia among BD patients is high. The presence of thrombophilia still affects the physician decision regarding the initiation and duration of anticoagulation in BD. The incidence of bleeding with long term anticoagulation is low. To best of our knowledge this is the first study to report solid cancer in Saudi BD patients. Further research is needed to find out the prevalence of cancer among BD in Saudis.

\section{Conflict of Interest}

The authors declared that there is no conflict of interest.

\section{Acknowledgments}

The authors are grateful to Mrs. Maram Altuwaijri for her effort in data entry and Mr. Abdelmoneim Eldali for his effort in the statistical analysis of our study.

\section{References}

[1] Mizuki N, Meguro A, Tohnai I, et al.: Association of major histocompatibility complex class I chain-related gene A and HLA-B alleles with Behcet's disease in Turkey. Jpn J Ophthalmol 2007; 51: 431-436.

[2] Ando H, Mizuki. Identification of a novel HLA-B allele $(B * 4202)$ in a Saudi Arabian family with Behcet's disease. Tissue Antigens. 1997; 49 (5): 526-8. PMID: 9174150.

[3] Hamuryudan V, Er T, Seyahi E, et al. Pulmonary artery aneurysms in Behçet syndrome. Am J Med 2004; 117: 867.

[4] Tayer-Shifman OE. Major vessel thrombosis in Behçet's disease: the dilemma of anticoagulant therapy - the approach of rheumatologists from different countries. Clin Exp Rheumatol. 2012 Sep-Oct; 30 (5): 735-40.

[5] Al-Dalaan AN. Behçet's disease in Saudi Arabia. J Rheumatol. 1994 Apr; 21 (4): 658-61.

[6] Yazici Y, Yurdakul S, Yazici H. Behçet's syndrome. Curr Rheumatol Rep 2010; 12: 429-35.

[7] Sakane T, Takeno M, Suzuki N, Inaba G. Behçet's disease. N Engl J Med 1999; 341: 1284-91.

[8] Xiuhua Wu,, Guohua Li,, Xinxiang Huang,, Li Wang,, Wanli Liu, Behçet's Disease Complicated with Thrombosis-A Report of 93 Chinese Cases. Medicine (Baltimore). 2014 Dec; 93 (28): e263.

[9] Erkan Alpsoy. New Evidence-Based Treatment Approach in Behçet's Disease. Pathology Research International. Volume 2012 (2012), Article ID 871019, 11 pages.

[10] Sarr SA, Superior vena cava syndrome revealing a Behçet's disease. Thromb J. 2015 Jan 31; 13: 7.

[11] Saadoun D, Wechsler B. Behcet's disease. Orphanet J Rare Dis. 2012; 7: 20. 
[12] Calamia KT, Schirmer M, Melikoglu M. Major vessel involvement in Behcet's disease: an update. Curr Opin Rheumatol. 2011; 23: 24-31.

[13] R. J. Ames, A. Steuer, A. Pap, and A. M. Denman, "Thrombosis in Behcet's disease: a retrospective survey from a single UK centre," Rheumatology; 40 (6): 652- 655, 2001.

[14] Emire Seyahi, MD, Melike Melikoglu, Canan Akman, Vedat Hamuryudan, Harun Ozer, Gulen Hatemi, Sebahattin Yurdakul, Hasan Tuzun, Buge Oz, and Hasan Yazici, "Pulmonary Artery Involvement and Associated Lung Disease in Behçet Disease", Medicine 2012; 91: 35-48.

[15] Micaela La Regina, Armen Yuri Gasparyan, Behçet's Disease as a Model of Venous Thrombosis. Open Cardiovasc Med J. 2010; 4: 71-77.

[16] NŞ, Salgür F, Cansu DÜ, et al. Combined thrombophilic factors increase the risk of recurrent thrombotic events in Behcet's disease. Clin Rheumatol 2010; 29: 1367-1372.

[17] Puja Mehta, Thrombosis and Behçet's syndrome in nonendemic regions. Is there a way around? Rheumatology (2010) 49 (11): 2003-2004.
[18] Alibaz-Oner F, et al. Study: No benefit from anticoagulant use in Behçet's disease. Medicine. 2015.

[19] Giacomo Emmi, Elena Silvestri, Danilo Squatrito. Thrombosis in vasculitis: from pathogenesis to treatment. Thromb J. 2015; 13: 15 .

[20] Kural-Seyahi E, Fresko I, Seyahi N, et al. The long-term mortality and morbidity of Behçet syndrome: a 2-decade outcome survey of 387 patients followed at a dedicated center. Medicine (Baltimore) 2003; 82: 60.

[21] Saadoun D, Wechsler B, Desseaux K, et al. Mortality in Behçet's disease. Arthritis Rheum 2010; 62: 2806.

[22] Kaklamani VG, Tzonou A, Kaklamanis PG. Behçet's disease associated with malignancies. Report of two cases and review of the literature. Clin Exp Rheumatol. 2005 Jul-Aug; 23 (4 Suppl 38): S35-41.

[23] Lin Y, Li G, Zheng W, Tian X, Zhang F. Behcet's disease associated with malignancy: a report of 41 Chinese cases. Int J Rheum Dis. 2014 May; 17 (4): 459-65. 